\title{
Tipos de encuestas considerando la dimensión temporal
}

\section{Vidal Díaz de Rada}

Universidad Pública de Navarra. Departamento de Sociología

Campus de Arrosadía s/n. 31006 Pamplona

vidal@unavarra.es

\section{Resumen}

Presentamos un trabajo sobre la encuesta, uno de los procedimientos más populares de recogida de datos desde el gran desarrollo de la investigación de opinión y mercados en la sociedad americana de los años cuarenta. El punto de partida que da origen a este trabajo es una inquietud, manifestada por García Ferrando y compartida totalmente por el que escribe estas líneas, cuando señala que «diversos aspectos centrales de la investigación mediante encuestas [...] han sido escasamente investigados, o por lo menos no lo suficiente como el amplio uso de encuestas merece». Nuestro objetivo se centra en realizar una diferenciación de las encuestas considerando el análisis de la dimensión temporal y presentando una tipología de los tipos de encuesta según este criterio.

Palabras clave: encuestas longitudinales, diseños prospectivos, estudios retrospectivos, encuestas de tendencias, encuestas de cohorte, encuestas de panel.

\section{Abstract. Longitudinal research and survey research}

The first part of the paper reviews the abilities of alternative survey design across time: prospective design, retrospective studies, cross-sectional surveys, cohort surveys, panel survey. The second part concentrates in panel surveys. It discusses the decision and need to be made in design a panel survey, the problem of nonresponse, time in sample bias and the seam effect, and some problems.

Key words: longitudinal research, prospective design, retrospective studies, cross-sectional surveys, cohort surveys, panel survey.

\section{Sumario}

1. El estudio de la dimensión temporal en la investigación con encuesta

2. Tipología de los diseños longitudinales: criterios definitorios

3. Diseños considerando la dimensión temporal
4. Ventajas del panel

5. Pertinencia de las críticas recibidas por el panel

Bibliografía 
El análisis de la literatura sobre métodos de investigación destaca la versatilidad y la adecuación de la encuesta como herramienta de investigación social; afirmación que, en numerosas ocasiones, se ha utilizado para legitimar el empleo de la encuesta en el análisis de cualquier problema social. Esto ha producido una utilización «abusiva» de esta técnica, al ser empleada para conocer cualquier aspecto de la realidad, sin considerar la naturaleza del objeto de estudio. Uno de los motivos de esta situación puede venir del desequilibrio existente entre la gran importancia de la encuesta en la investigación social y el escaso número de trabajos que se publican en nuestro país sobre los efectos, las limitaciones y las ventajas de esta técnica de recogida de información (Sánchez Carrión, 1996: 128-9). En un trabajo publicado hace ya unos años (Díaz de Rada, 2001: 7), mostrábamos nuestro asombro por esta situación, sin embargo cuatro años más tarde no creemos que la situación haya cambiado sustancialmente, aunque la utilización de la encuesta en la investigación actual se ha incrementado sustancialmente: las 7.855.000 encuestas realizadas por las empresas pertenecientes a AEDEMO y ANEIMO que se realizaron en 1998 han aumentado hasta las 9.072.000 en el año 2001 ${ }^{1}$ (Alós, 2002: 77).

Con este trabajo tratamos de contribuir a paliar tal desequilibrio, al presentar una clasificación de los tipos de encuestas según la dimensión tempo$\mathrm{ral}^{2}$. El punto de partida que da origen a este trabajo es una inquietud, manifestada por García Ferrando y compartida totalmente por el que escribe estas líneas, cuando señala que «diversos aspectos centrales de la investigación mediante encuestas [...] han sido escasamente investigados, o por lo menos no lo suficiente como el amplio uso de encuestas merece» (García Ferrando, 2000: 198).

Debe tenerse en cuenta que la exposición de los diferentes tipos de encuestas se realizará poniendo de manifiesto los problemas propios de cada una. Es preciso señalar que estos problemas se inscriben dentro del contexto general de la investigación mediante encuesta, de modo que dejamos fuera las críticas a la técnica de la encuesta - críticas comunes a todos los diseños presentados- para centrarnos únicamente en las mejoras de un diseño sobre otro. Las críticas a la encuesta aparecen en los trabajos sobre los fundamentos operativos de esta técnica, por lo que se aconseja al lector interesado la consulta de un trabajo anterior (Díaz de Rada, 2001).

\section{El estudio de la dimensión temporal en la investigación con encuesta}

Considerando la dimensión temporal de los fenómenos analizados, los estudios no experimentales (correlacionales) realizados con encuestas se dividen en transversales y longitudinales. El estudio transversal se caracteriza porque

1. No se ofrecen cifras más recientes porque después del año 2002 no se ha vuelto a realizar el estudio.

2. Para referirnos a las investigaciones formadas por cada tipo de encuesta, emplearemos la palabra "diseño", fundamentalmente porque la elección del tipo de encuesta se realiza en la fase de planificación y diseño de la investigación. 
la recogida de información se realiza mediante una única medición. Se trata de estimaciones estáticas que corresponden a una fotografía de la realidad en un momento concreto, a una instantánea que recoge una situación estática.

Estos diseños se utilizan fundamentalmente para llevar a cabo estimaciones descriptivas del objeto de estudio, aunque también permiten realizar explicaciones al efectuar comparaciones entre subgrupos. Dividiendo la muestra en subgrupos y buscando similitudes y diferencias en la variable dependiente, es posible conocer la influencia de ciertas variables.

Sin embargo, este procedimiento tan sólo permite detectar la relación entre una serie de variables y el término dependiente, siendo incapaz de precisar cómo las variables independientes determinan las variaciones en el término dependiente ${ }^{3}$. Esta imposibilidad para detectar relaciones causales, es el mayor problema de los diseños transversales. Sin embargo, el diseño transversal es uno de los más utilizados en la investigación social por su capacidad para hacer inferencias, por la facilidad y la economía en su ejecución, y por la rapidez a la hora de proporcionar resultados. En el cuadro 1, se presentan los principales rasgos de los diseños transversales y longitudinales.

Otra ventaja de las encuestas transversales es su sencillez para investigar relaciones complejas entre grandes conjuntos de variables y sujetos (Kish, 1965: 476). A esto hay que añadir que una fecha específica de referencia puede adecuarse mejor a determinados requisitos legales o tradicionales (de comparación con otras fuentes). Por último, otra de las razones que explican esta elevada utilización es su adecuación para el estudio de fenómenos estables en el tiempo.

Si la situación analizada no es muy estable, será necesario utilizar diseños longitudinales caracterizados por recoger información en distintos momentos temporales. En este tipo de diseños, el objeto de estudio es observado repetidamente durante un intervalo de tiempo, con varias administraciones del mismo instrumento a los mismos elementos (o a elementos comparables), y tiene como objetivo la descripción y explicación de los procesos producidos como consecuencia del tiempo transcurrido entre una medición y otra (Ruspini, 1999: 221).

Estos diseños plantean una similitud con el método experimental, que, por otra parte, es el único que permite determinar relaciones causales ${ }^{4}$. Recordemos que, en su formato más sencillo, los diseños experimentales realizan una medi-

3. Es decir, las investigaciones transversales no permiten realizar explicaciones causales —objeto preferente del conocimiento científico-, puesto que no cumplen los requisitos básicos que precisa toda explicación causal:

1. Relación con un único sentido donde la variable dependiente $Y$ varía según los valores de la variable independiente $X$;

2. la causa debe ser anterior que el efecto, siendo necesario conocer el momento de aparición de cada una;

3. esta relación implica que toda la variabilidad de la variable dependiente $Y$ esté explicada por la variable independiente $X$, es decir, que no hay otras variables que afecten a esa relación, bien individualmente o de forma conjunta con la variable $X$.

4. Unico método que es capaz de asegurar que la relación entre dos variables es real y no espúrea» (Alvira y otros, 1981: 58). 
Cuadro 1. Ventajas e inconvenientes de los diseños transversales y longitudinales.

\section{Diseño transversal}

Objetivo: describir la realidad en un momento determinado.

Ventajas:

1. Facilidad para hacer inferencias (alta validez externa).

2. Sencillez y economía en su ejecución, especialmente con muestras pequeñas y diseminadas.

3. Proporcionan resultados con gran rapidez.

4. Sencillez para investigar relaciones complejas entre grandes conjuntos de variables y sujetos.

5. Una fecha específica de referencia se adecua mejor a requisitos legales o tradicionales.

6. Muy adecuada para situaciones estables en el tiempo, por su sencillez $-\mathrm{y}$ economía - en la recogida de datos.

Inconvenientes:

1. Incapacidad para revelar relaciones causales. Baja validez interna.

2. Ausencia de medidas en diversos puntos cronológicos.

\section{Diseño longitudinal}

Objetivo: detectar el cambio de la situación producto del tiempo. Para ello, recogen información en diferentes momentos temporales.

Ventajas:

1. Alta validez interna.

2. Permite explicar las causas de los fenómenos.

3. Detecta la presencia de efectos provocados por el momento de medición (temporalidad), la maduración (edad) y la cohorte de pertenencia.

Inconvenientes

1. Encarecimiento del estudio fruto de las mediciones realizadas.

2. Se precisa mucho tiempo para disponer de la información.

ción inicial, suministran un estímulo o efectúan una intervención en la realidad, y posteriormente llevan a cabo una segunda medición. La diferencia entre ambas mediciones se atribuye al estímulo suministrado. Esta situación es fácilmente reproducible en un diseño longitudinal, si bien añade la ventaja que el investigador puede analizar la influencia de ese estímulo no en un ambiente controlado de laboratorio, sino en la realidad. De hecho, cuando un estudio longitudinal realiza la recogida de información antes y después de un suceso concreto, no hace sino reproducir esta situación experimental.

Pese a la facilidad del experimento para determinar relaciones causales, la artificialidad de las situaciones empleadas y su baja validez externa explican su escasa utilización en sociología. Frente a esta situación, los diseños longitudinales utilizan la lógica del experimento en una situación real y con datos que permiten realizar inferencias. Este hecho explica el gran auge de los diseños longitudinales y la escasa utilización del experimento en la investigación actual. 
Como contrapartida, el elevado número de mediciones aumenta notablemente el coste del estudio (cuadro 1); incremento que dista mucho de ser proporcional (esto es, un estudio longitudinal con tres mediciones no cuesta el triple que un estudio transversal). Además, no todos los estudios longitudinales precisan realizar mediciones en momentos distintos. Existe un tipo de diseño longitudinal, conocido como diseño retrospectivo o diseño transversal ordenado en el tiempo, que, de una sola vez, realiza consultas referidas al pasado y al presente de los sujetos investigados. Las características de estos diseños serán expuestas seguidamente, dentro de la explicación de los criterios definitorios para la elección de un diseño longitudinal.

\section{Tipología de diseños longitudinales: criterios definitorios}

Expondremos a continuación los aspectos que deben ser considerados en la elección de un diseño longitudinal, aspectos que proporcionarán una tipología de los distintos tipos de diseños para medir la influencia del tiempo. Es evidente que el empleo de un diseño longitudinal concreto está determinado por el objeto de estudio; ahora bien, la elección del tipo de diseño a utilizar dependerá de la consideración de tres elementos:

1. En primer lugar, esta elección comienza considerando si la información va a ser recogida en uno o en varios momentos temporales. Aunque el diseño longitudinal se define por recoger información referida a dos momentos distintos, es posible obtener esta información de una sola vez utilizando un diseño retrospectivo. Este diseño recoge información relativa a momentos pasados y presentes de la población objeto de estudio, en base a la memoria e información proporcionada por los propios entrevistados. La comparación entre la situación presente y la pasada da una idea del efecto del tiempo.

La gran ventaja de este diseño, frente al prospectivo, es la rapidez en la obtención de la información y el escaso costo económico del mismo. Sin embargo, presenta algunos problemas provocados por los fallos en la memoria de los entrevistados, fallos que serán más acentuados en la medida que se pregunta sobre cuestiones muy lejanas o poco importantes en la vida de los individuos. La similitud metodológica de la investigación retrospectiva con los diseños transversales nos lleva a centrar nuestra exposición en los diseños prospectivos; que son los más adecuados para estudiar el efecto del tiempo.

2. Satisfecho el primer requisito, y siempre que se pretenda realizar varias mediciones, el investigador debe plantearse si van a utilizarse las mismas unidades durante todo el proceso de medición o si se preguntará sobre los mismos asuntos a personas distintas. La medición repetida de las mismas personas en diversas ocasiones recibe el nombre de diseño panel, mientras que la utilización de personas distintas se denomina diseño de tendencias.

3. El tercer requisito está relacionado con la amplitud de la población objeto de estudio: ¿¿Se trata de estudiar a toda la población, o tan sólo a un grupo 
específico? Cuando se estudia un grupo de población, un grupo delimitado por haber presenciado un determinado elemento o haber nacido entre unas fechas, nos encontramos con estudios de cohorte.

La consideración conjunta de estos aspectos proporciona cuatro tipos de diseños que serán explicados en el próximo apartado: diseño de tendencias, cohorte de tendencias, diseño de panel y cohorte de panel.

\section{Diseños considerando la dimensión temporal}

Vistos los aspectos básicos que caracterizan a cada diseño, pasaremos a presentar los diferentes tipos de diseños longitudinales. A excepción del diseño retrospectivo, el elemento común a todos los diseños longitudinales es que el instrumento de medida es el mismo y se aplica de la misma forma en cada medición. Ahora bien, atendiendo al número de mediciones efectuadas en cada unidad muestral, es posible distinguir entre diseño longitudinal de tendencias y de panel.

\section{Diseño de tendencias}

Considerando que el objeto de estudio de los diseños longitudinales es detectar como varían una serie de aspectos a lo largo del tiempo (cuadro 1), los diseños de tendencias se caracterizan por realizar las mediciones a muestras diferentes en varios momentos temporales. Su elemento definitorio es la elección de una muestra distinta para cada medición, si bien se busca que éstas presenten características similares en cuanto a sus rasgos sociodemográficos. Un ejemplo de estudio de tendencias son los barómetros del Centro de Investigaciones Sociológicas, que repiten periódicamente una serie de preguntas referidas a la situación política y económica del país. Debe tenerse en cuenta que el instrumento de medida es el mismo, y que la muestra cambia cada mes.

El diseño de tendencias es idóneo cuando se trata de investigaciones que van a realizarse durante un largo período de tiempo, se pretende efectuar mediciones muy espaciadas en el tiempo y el objeto de estudio está formado por estratos poblacionales con una gran movilidad geográfica. Comparado con el diseño transversal, los estudios de tendencias presentan varias ventajas que aparecen sintetizadas en el cuadro 2 (Kish, 1965: 475-476):

a) En primer lugar, permiten aumentar la calidad de los resultados obtenidos, reducir costes, o ambos conjuntamente. Llevar a cabo la selección, contratación y adiestramiento de un equipo de personas para una única investigación supone un elevado coste económico, coste que se rentabiliza mejor cuando ese personal es utilizado en varios estudios.

b) En segundo lugar, las muestras repetidas pueden producir estadísticas de series cronológicas que permitan estimar cambios y tendencias (estacionales, anuales, etc.) y detectar variaciones irregulares y repentinas. 
c) En tercer lugar, la unión de encuestas repetidas durante un período de tiempo proporciona una inferencia más precisa que la basada en muestras únicas, al eliminar las variaciones irregulares a las que están expuestos los diseños transversales.

La mayor parte de las veces, los estudios de tendencias no se elaboran específicamente para analizar el efecto del tiempo, sino que más bien se trata de estudios transversales - realizados con unos fines determinados- que forman una serie cronológica que, en un segundo momento, será aprovechada para un análisis de tendencias (como sucede, por ejemplo, en los barómetros del CIS y en las series cronológicas que distribuye este centro ${ }^{5}$ ). Así, la gran ventaja del diseño de tendencias, desde nuestro punto de vista, es la facilidad para formar series cronológicas - y así generar descripciones evolutivas - llevando a cabo análisis secundarios de encuestas realizadas por diversos investigadores.

En relación con el panel, que será analizado más adelante, el diseño de tendencias presenta varias ventajas:

1. Al elegir una nueva muestra cada vez que se realiza una medición, el diseño de tendencias no presenta abandonos entre una medición y otra.

2. La elaboración de nuevas muestras en cada medición evita la «búsqueda» de los sujetos seleccionados anteriormente.

3. En tercer lugar, este diseño permite una mayor flexibilidad en el diseño de la investigación, puesto que posibilita diseñar muestras más amplias de determinados subgrupos, introducir nuevos temas, etc. (Hakim, 2000: 96).

4. Como cuarta ventaja, la administración de un sólo cuestionario a cada sujeto evita los efectos adulterantes producidos por la entrevista anterior. En el estudio de panel hay que considerar que, después de una primera entrevista, las personas tienen más conocimiento sobre el tema de estudio, pueden reflexionar sobre él, al tiempo que prestan más atención a esa temática. Este hecho no ocurre en un estudio de tendencias.

5. Por último, hay una mayor precisión en la comparación de los grupos en el tiempo como consecuencia del mayor tamaño de las muestras utilizadas en los diseños de tendencias.

Existe un caso particular de diseño de tendencias, conocido como «diseño longitudinal de cohorte», que selecciona una o varias cohortes de la misma población y que sigue el desplazamiento de éstas a lo largo del tiempo. Al ser un caso particular del diseño de tendencias, se realiza una única medición en cada entrevistado y se observan distintos individuos pertenecientes a la misma cohorte en momentos diferentes (Buck y otros, 1996: 5).

5. Ver, por ejemplo, los estudios distribuidos en CD-ROM por el CIS: Estudios postelectorales de las elecciones autonómicas en el País Vasco (1984-1998); Estudios postelectorales de las elecciones autonómicas en Galicia (1981-1997); Estudios postelectorales de las elecciones generales (1982-1996); Opiniones y actitudes de los españoles, etc. (Centro de investigaciones Sociológicas, 1995). 
Cuadro 2. Ventajas del diseño longitudinal de tendencias.

\section{Resulta apropiado cuando (condiciones de utilización):}

1. Se trata de investigaciones que van a realizarse durante un largo período de tiempo;

2. efectuando mediciones muy espaciadas en el tiempo, y

3. el objeto de estudio está formado por estratos poblacionales con una gran movilidad.

\section{Ventajas frente al diseño transversal:}

1. Permiten aumentar la calidad de los resultados obtenidos, debido al mayor aprovechamiento de los recursos dedicados a la selección y formación del personal de recogida de información.

2. Producen estadísticas de series temporales que facilitan la estimación de cambios y tendencias, lo cual permite detectar variaciones irregulares y repentinas.

3. La unión de encuestas repetidas durante un período de tiempo proporciona inferencias estadísticas más precisas que la basada en muestras únicas.

4. Facilidad para formar series cronológicas realizando análisis secundarios de fuentes de datos recogidas por otros investigadores.

\section{Ventajas frente al diseño de panel:}

1. No hay abandonos entre una medición y otra.

2. Al utilizar una muestra cada mes, se evita realizar una búsqueda y localización de los sujetos ya seleccionados.

3. Mayor flexibilidad en el diseño de la investigación por la posibilidad de introducir nuevos temas en el cuestionario, cambiar el diseño muestral, el procedimiento de recogida de información, etc.

4. Llevar a cabo una única medición evita determinados efectos producidos por la entrevista anterior (reactividad a las mediciones previas, efecto del pretest).

5. Mayor precisión como consecuencia del mayor tamaño de las muestras.

En el diseño de cohorte, el objeto de estudio se circunscribe a una parte de la población, una cohorte, definida como un grupo de individuos que han compartido una misma experiencia en un determinado período temporal (Glenn, 1977: 8). Forman parte de la misma cohorte los nacidos en el mismo mes - o en el mismo año-, todos los que han terminado sus estudios superiores en un momento concreto, los que se casaron o se divorciaron en el mismo año, etc. Tras la definición de la cohorte, se analiza su evolución realizando la medición de una —o varias - cohortes en dos o más momentos temporales. El objetivo de este diseño es localizar los cambios que el transcurso del tiempo ha producido en la variable dependiente, y si dichos cambios afectan por igual a todos los pertenecientes a la misma cohorte. Esta información se obtiene considerando por separado la influencia de la edad, la cohorte de pertenencia y el momento de medición.

Los pertenecientes a cada una de estas cohortes han nacido y se han socializado en un ambiente concreto, con unas determinadas influencias sociales, políticas y económicas. Esto es, cada cohorte ha experimentado unos sucesos en un momento determinado de su vida, sucesos diferentes a los que experi- 
mentó la cohorte anterior en esa misma edad. Es muy probable que los nacidos en 1990 no recuerden — dentro de unos años- qué sucedió el 11 de septiembre del 2001, mientras que otras cohortes nacidas antes probablemente lo recuerden durante toda su vida.

\section{Diseño de panel}

Cuando el objetivo de la investigación es toda la población, pero en vez de realizar una única medición a cada entrevistado se realizan varias, estamos en presencia de diseños longitudinales de panel. El diseño de panel selecciona la muestra al principio de la investigación y lleva a cabo una recogida de datos sobre el mismo grupo de sujetos en varios momentos diferentes, con el objetivo de obtener cambios individuales (Kish, 1995: 223). En la medida que las personas seleccionadas son las mismas, y la diferencia es el tiempo que ha pasado entre una medición y otra, es posible conocer cómo determinadas circunstancias generan cambios en los comportamientos y en las actitudes de los individuos, lo que permite conocer con gran precisión las causas del cambio de determinadas conductas, opiniones, interpretaciones, etc.

Las principales diferencias respecto al resto de diseños son la periodicidad de las mediciones y la posibilidad de realizar un seguimiento continuado de los fenómenos objeto de estudio, lo que proporciona un conocimiento más profundo y detallado del comportamiento de los sujetos analizados. De hecho, Mayntz considera que mientras el diseño de tendencias se centra en la descripción del cambio, el objetivo del diseño de panel es, fundamentalmente, la explicación de éste (1976: 177). Otros objetivos del diseño de panel son (DeVaus, 2001: 114-118):

A. Describir las pautas de estabilidad y cambio.

B. Desvelar el orden temporal de los fenómenos.

C. Estudio de «transiciones», llegar a establecer análisis de trayectorias vitales, ocupacionales, etc.

D. Por último, conviene destacar su utilización para desvelar la incidencia de nuevos eventos entre dos o más fechas (Kish, 1995: 204).

Una síntesis de estas ventajas se muestran en el cuadro 3.

Terminaremos los tipos de diseños longitudinales señalando que la elección del tipo de investigación va a depender de la frecuencia de ocurrencia de

Cuadro 3. Características del diseño longitudinal de panel.

1. Describe pautas de estabilidad y cambio a niveles muy desagregados.

2. Permite conocer el orden temporal de los fenómenos.

3. Posibilidad de llevar a cabo análisis de trayectorias; siguiendo toda la vida de un individuo.

4. Muy utilizado para desvelar la incidencia de nuevos eventos entre dos (o más) momentos temporales. 
los fenómenos y de su duración. Así, Buck y otros (1996: 2) señalan que «si se analizan hechos con baja frecuencia de ocurrencia y gran duración, la mejor solución es utilizar diseños transversales. Por otro lado, cuando el objetivo es obtener información sobre sucesos con alta ocurrencia y escasa duración, es mejor utilizar un panel» (1996: 2).

Resumiendo lo visto hasta ahora, los diseños transversales proporcionan información de un momento concreto; mientras que los diseños de tendencias informan de los cambios a través del tiempo. Los diseños de cohorte - por su parte- utilizan varios diseños de tendencias para describir los cambios agregados a través del tiempo e identificar la cantidad de este cambio, que es producido por el efecto de la medición, la edad y la cohorte (DeVaus, 2001: 211). Los estudios de panel, en los que me centraré a partir de este momento, proporcionan información con un mayor nivel de precisión, al distinguir entre el cambio individual y el grupal.

\section{Ventajas del panel}

Presentados los distintos diseños longitudinales y vistas las ventajas de unos sobre otros, es el momento de centrar la exposición en el diseño longitudinal de panel, exponiendo las ventajas - y los inconvenientes - del panel frente a los diseños anteriores (cuadro 4). La investigación de panel tiene su origen en el ámbito del consumo y de la opinión pública con los trabajos de Lazarsfeld y colaboradores en la década de 1930, y hoy se aplican en un gran número de áreas de investigación, como el análisis de la preferencia política, los estudios de trayectoria ocupacional y salarial, los hábitos de consumo, la investigación comercial, los medios de comunicación de masas, el conocimiento de opiniones y actitudes, etc.

La principal ventaja es la posibilidad de detectar, además de los cambios agregados netos proporcionados por los diseños anteriores, los cambios referidos a nivel de los elementos de una población, los cambios brutos (Kalton, 1983: 53).

Otra de las grandes ventajas del panel es su utilidad para conocer la dinámica del cambio, puesto que detecta las circunstancias en las que se producen determinados acontecimientos. Este diseño permite desvelar las condiciones en las que la gente cambia su comportamiento político, sus pautas de consumo, etc. Por este motivo, el panel es utilizado frecuentemente para «seguir el rastro» de la evolución del comportamiento de los consumidores, buscando variaciones actitudinales, cambios de pautas de conducta sobre modas, etc. Analizando las características de las personas que cambian y los factores que lo justifican, es posible realizar predicciones sobre la dinámica del cambio.

La ventaja anterior genera que el panel puede ser utilizado para medir las situaciones de cambio, así como su dirección, cuando se introduce una variable experimental. El hecho de poder controlar a los entrevistados y comprobar como reaccionan ante un estímulo, permite una mejor comprensión del proceso de cambio. Este diseño puede, así, utilizarse para conocer la influen- 
Cuadro 4. Ventajas del diseño longitudinal de panel.

\section{Ventajas respecto a los diseños anteriores:}

1. Es el único diseño que permite medir los cambios a nivel individual (bruto) y agregado (neto).

2. Conocimiento de la dinámica del cambio, detectando las circunstancias en las que se producen determinados acontecimientos.

3. Permite conocer la reacción a una variable experimental.

4. Agregando nuevas preguntas en las siguientes mediciones, es posible lograr gran información sin incrementar el coste.

5. Reducción de errores al posibilitar — con un bajo coste- utilizar verificaciones y controles en las respuestas.

6. Realizar varias entrevistas con los entrevistados permite una cierta "familiaridad» que se traduce en una mejora en la calidad —y cantidad — de la información recogida.

cia de la propaganda en el voto político, el cambio de imagen de una institución tras poner en marcha una campaña promocional, el efecto de ciertos eventos de opinión pública y campañas mediáticas, etc. De hecho, ésta es una de las situaciones más apropiadas para analizar con un panel, como señaló Lazarsfeld en uno de los primeros trabajos publicados sobre el tema (1993: 147-148, e. o. 1948).

El panel realiza una única medición de las variables sociodemográficas, puesto que, al tratarse de los mismos individuos, es posible «obtenerlas» de los registros anteriores. Únicamente el cuestionario de la primera medición incluye preguntas sociodemográficas, de modo que, en los cuestionarios sucesivos, éstas se eliminan o son sustituidas por otras. Agregando nuevas preguntas en las oleadas sucesivas, es posible obtener más información sobre cada entrevistado sin incrementar el coste.

Este diseño consigue, además, reducir los errores, al utilizar diversos procedimientos para verificar las diferencias y las consistencias ${ }^{6}$. Asimismo, facilita la posibilidad de emplear gran número de verificaciones y controles para prevenir posibles sesgos. Al tratarse de un cuestionario que se repite en el tiempo, tampoco supone un costo excesivo la creación de edits y consistencias lógicas entre preguntas (Kish, 1995: 238-239).

Por último, el hecho de realizar varios contactos con los mismos entrevistados, genera una cierta "familiaridad» que ayuda a disminuir los recelos iniciales sobre la encuesta, lo que permite obtener información de más calidad en las entrevistas sucesivas (Buck y otros, 1996: 24).

6. Por ejemplo, utilizar una encuesta telefónica para consultar las inconsistencias de la entrevista personal. Así opera, por ejemplo, el Panel de Consumidores (Pueyo, 1996: 11). 


\section{Pertinencia de las críticas recibidas por el panel}

Comparado con los diseños anteriores, el diseño de panel presenta también varios inconvenientes. Concluiremos esta exposición con una recopilación exhaustiva de todas las desventajas atribuidas a este diseño y realizando un balance del grado de solidez de tales imputaciones. Esta relación de desventajas va a estar dividida en dos partes: en la primera se recogen las relacionadas con el muestreo y en la segunda, aquéllas referidas a la calidad de las mediciones efectuadas.

1. Respecto a las relacionadas con el muestreo, la primera crítica señala la dificultad para seleccionar personas que colaboren durante un largo período de tiempo. En Estados Unidos y en el Reino Unido, los rechazos en la primera visita oscilan entre el 20 y el 30\% (Kish, 1995: 236; Buck y otros, 1996: 8); cifras que no considero elevadas cuando se analiza las tasas de "no respuesta» en encuestas transversales en nuestro país (Díaz de Rada, 2000).

2. Un efecto directo de la desventaja anterior es la falta de representatividad de las muestras, debido a que las personas que participan en los paneles no suelen representar a una selección aleatoria del universo poblacional, porque no todo el mundo acepta colaborar en un panel. Pudiera decirse que existe un sesgo de representatividad de la muestra debido a la autoselección de los entrevistados. Sin embargo, cuando se consideran las características de los que más cooperan en el panel, se aprecia una coincidencia con las personas que más colaboran en encuestas transversales, de modo que - a nuestro juicio- se trata de un problema de la técnica de la encuesta en muestreos aleatorios, y no tanto un problema de un tipo concreto de encuesta.

3. En tercer lugar, destacar el desgaste de la muestra debido a las molestias causadas por las sucesivas mediciones, que se traducen en abandonos a lo largo de la investigación. Este abandono puede tener causas naturales (fallecimiento del panelista), si bien, en su mayor parte, se produce por otros motivos, como pérdida de interés por el proyecto, falta de respuesta, dificultad de relocalización por cambios de domicilio, etc.

Como la mayor parte de las veces el abandono está originado por el cansancio que supone colaborar durante un largo período de tiempo, se busca evitar este abandono motivando a los panelistas por medio de regalos, suscripciones a revistas, dinero en efectivo, etc. Junto a la utilización de esos incentivos, es muy importante crear una relación personal con los entrevistados, con el fin de promover - y aumentar así- su interés en la investigación. Un buen ejemplo es el Estudio de Desarrollo de la Infancia — NCDS (National Child Development Study)_, que envía felicitaciones de cumpleaños a sus 17.000 entrevistados, y el Estudio de Dinámica del Gasto -PSID (Panel Study of Income Dynamics)—, que envía resúmenes de los resultados (Hakim, 2000: 124). Estas estrategias permiten, a su vez, conocer los cambios de domicilio antes de llevar a cabo la siguiente medición. 
Sin embargo, hay ocasiones en las que estas estrategias no proporcionan los resultados esperados, de modo que es necesario utilizar otras, como son el panel simple con reemplazamiento, y el panel rotatorio. En el primero, los abandonos son «repuestos» por nuevos miembros similares a los que han salido; mientras que el panel rotatorio realiza — tras cada medición- un cambio de los entrevistados más antiguos, procedimiento conocido también como "rotación de la muestra con solapamiento parcial» (Azorín y Sánchez-Crespo, 1986: 233-235).

4. El problema anterior hace difícil que se cumpla un requisito básico para el funcionamiento del panel, como es la necesidad de realizar las siguientes mediciones sobre las mismas personas. No obstante, investigaciones recogidas por Miller señalan que, para que esta técnica funcione de forma efectiva, «basta con que, al menos, permanezca un $67 \%$ de la muestra original» (1991: 171). De hecho, la Encuesta de Población Activa (EPA) española mantiene constante el 83\% de la muestra, y la EPA norteamericana (Current Population Survey), el 75\%.

El segundo grupo de críticas están relacionadas con la calidad de las mediciones realizadas, el instrumento de medida.

5. Comenzaremos con la crítica que advierte de la percepción — por parte de los entrevistados- que las encuestas son menos anónimas que las realizadas en el resto de diseños (Mayntz y otros, 1976: 193). La utilización de encuestas personales asistidas por ordenador (CAPI), que «recuerdan» las respuestas anteriores, contribuyen aún más a incrementar esta falta de anonimato. Es preciso tratar con sumo cuidado este tema, enviando cartas y comunicaciones a los entrevistados para recordarles la absoluta confidencialidad de la información aportada. De hecho, las encuestas postales tampoco son anónimas y las personas responden muy sinceramente a determinadas conductas «socialmente mal vistas», como el consumo de alcohol y drogas, infidelidades en el matrimonio, cantidad de dinero gastada en juegos de azar, etc.

6. En sexto lugar, la imposibilidad de esta técnica para captar la aparición de nuevas cualidades en el seno de los grupos que estudia, puesto que las siguientes mediciones se realizan respecto a las mismas propiedades, es decir, aplicando de nuevo el instrumento de medida de la primera medición (Mayntz y otros, 1976: 178). No podemos considerar adecuada esta crítica, puesto que, en la actualidad, numerosos paneles incorporan, junto al bloque de preguntas fijas, una serie de preguntas que varían cada mes (Miller, 1991: 168; Buck y otros, 1996: 23). Al estar colocadas al final, es de suponer que éstas no afectan al resto de preguntas del cuestionario.

7. La séptima crítica reseña los problemas de validez provocados por la reactividad del retest, sensibilización y aprendizaje. Las personas saben que están siendo observadas, de modo que el propio proceso de medición puede estar generando un cambio en el comportamiento habitual de estas personas (Cantor, 1998: 29-30; Lazarsfeld, 1993: 153). Miller (1991: 171) y Lazarsfeld (1993: 153) restan importancia a estos problemas, puesto que 
pueden ser cuantificados — y así eliminados — mediante un diseño de investigación que divida la muestra del panel en dos grupos: un grupo experimental y otro de control. Lazarsfeld (1993: 153) señala también que estos efectos desaparecen con las mediciones sucesivas, fundamentalmente a partir de la tercera. Más seria es la postura de Kish cuando destaca la escasa investigación realizada para detectar tales efectos, que contrasta con «unos recelos incongruentes muy generalizados» (1995: 237).

8. En octavo lugar se señala la deficiente fiabilidad de las mediciones por la influencia del ambiente en la entrevista, fallos en el entrevistador, en la codificación, etc. Mayntz y otros (1975: 194) señalan que la presencia de estos errores en el resto de encuestas es menor, puesto que - al ser aleatorias - se neutralizan recíprocamente, mientras que en el panel pueden provocar cambios ficticios.

Desde nuestro punto de vista, esto no es una desventaja, puesto que un estudio panel permite emplear una mayor cantidad de recursos para la selección y formación de un entrevistador que colaborará durante mucho tiempo en una misma investigación. Además, diversos estudios han demostrado que mantener la continuidad del entrevistador, es decir, que un panelista sea entrevistado siempre por el mismo entrevistador, consigue un notable aumento en la tasa y calidad de la respuesta: «la familiaridad con las unidades de muestreo y con los individuos puede tener a menudo resultados positivos, en contraposición a los resultados negativos del desgaste, la reactividad y el relajamiento" (Kish, 1995: 239). Por otro lado, numerosas investigaciones señalan que la realización de entrevistas sucesivas permite a las unidades seleccionadas responder mejor (Pueyo, 1996: 2; Barranco Sáiz, 1990: 37).

9. Como novena crítica, el relajamiento de las entrevistas repetidas puede generar un determinado sesgo. Los entrevistados pueden recordar $-\mathrm{y}$ repetir - la respuesta emitida en la medición anterior, aún sin pretenderlo. Los entrevistadores pueden bajar la guardia y cometer más errores. La utilización de paneles rotatorios disminuye notablemente el «efecto inercia» de los entrevistados, aunque las investigaciones de Kish no llegan a localizar tal sesgo (1995: 238). Sesiones de formación previas a cada oleada pueden reducir los errores de los entrevistadores.

Expuesta la pertinencia de estos inconvenientes, y confirmada la utilidad del panel para conocer el cambio, no acabamos de entender la escasa utilización del panel en nuestro país. Mucho más cuando — como hemos constatado- la mayor parte de las críticas recibidas desaparecen cuando se analizan en profundidad, lo que me lleva a considerar que se trata más bien de prejuicios que de desventajas reales. Terminaremos con una cita de Leslie Kish, como una especie de homenaje a su reciente fallecimiento: «debido a sus singulares ventajas, los paneles están poniendo de relieve resultados no descubiertos por otros métodos, pero no se emplean con mucha frecuencia debido a los difíciles problemas que van asociados a su utilización» (Kish, 1995: 234). 


\section{Bibliografía}

Alós, J. (2002). «Industria de los estudios de mercado en España 2001». Investigación y Marketing, vol. 76, p. 76-78.

Alvira Martín, F.; Avia, M. D.; Calvo, R.; Morales, J. F. (1981). Los dos métodos de las Ciencias Sociales. Madrid: Centro de Investigaciones Sociológicas.

AZOrín, F.; SÁNCHEZ-CreSPO, J.L. (1986). Métodos y aplicaciones del muestreo. Madrid: Alianza.

BARRANCO SÁInZ, F. J. (1990). «El panel de consumidores». MK Marketing y Ventas, no 35, p. 36-39.

BuCK, N.; ERMISCH, J.; HENKINS, S. P. (1996). Choosing a longitudinal surveys design: the issues. Occasional Paper of the ESRC Research Center on Micro-Social Change. Occasional Paper 96-1. Colchester: Universidad de Essex.

CANTOR, D. (1989). "Substantive implications of longitudinal design features: the National Crime Survey as a case study». En: KASPRZYK, D.; DunCAN, G. J.; Kalton, G.; Singh, M. P. Panel surveys. Nueva York: Wiley, p. 25-51.

CENTRO DE InVESTIGACIONES SOCIOlÓGICAS (1995). Estudios en CD-ROM. Consultado en: <http://www.cis.es/Page.aspx?OriginId $=68>$.

Díaz DE RADA, V. (2000). Problemas originados por la no respuesta en investigación social: Definición, control y tratamiento. Pamplona: Universidad Pública de Navarra.

- (2001). Diseño y elaboración de cuestionarios para la investigación comercial. Madrid: Esic.

García Ferrando, M. (2000). «La encuesta». En: García Ferrando, M.; IbáÑez, J.; ALVIRA, F. (eds.), El análisis de la realidad social. Madrid: Alianza, 3a ed., p. 167-201. (Edición original de 1986.)

GLEnN, N. D. (1977). Cohort analysis. Sage University Paper Series on Quantitative Applications in the Social Sciences, 07-05. Newbury Park, CA: Sage.

HAKIM, C. (2000). Research design: sucessful design for social and economic research. Londres: Routledge. (Edición original de 1987.)

KALTON, G. (1983). Introduction to survey samplig. Sage Univeristy Paper series on Quantitative Applications in the Social Sciences, vol. 35. Newbury Park, California: Sage.

KISH, L. (1965). Survey Sampling. Nueva York: Wiley. Versión española en 1965: Muestreo de encuestas. Méjico: Trillas.

- (1995). Diseño estadístico para la investigación. Madrid: Centro de Investigaciones Sociológicas. Monografía número 146.

LAZARSFELD, P. L. (1993). «The use of panels in social reseach». En: BoudON, R. Paul F. Lazarsfeld: On social research and its languaje. Chicago: University of Chicago Press, p. 147-157. Publicado originariamente en Proceedings of the American Philosophical Society, 1948, vol. 92, no 5, p. 405-410.

MAYNTZ, R.; HOLM, K.; HÜBNER, P. (1975). Introducción a los métodos de la sociología empirica. Madrid: Alianza.

Miller, D.C. (1995). Handbook of Research Design and Social Measurement. Londres: Sage.

PuEYO, S. (1996). "El panel de consumidores», apuntes del curso Investigación Mercadológica del Consumidor, curso 1995-96.

RUSPINI, E. (1999). «Longitudinal Research and the analysis of Social Change». Quality and Quantity, vol. 33, p. 219-227.

SÁNCHEZ CARRIÓn, J. J. (1996): «La calidad de la encuesta». Papers, 48, p. 127-146.

VAus, D. de (2001). Research Design in Social Research. Londres: Sage. 